\title{
Kollektive Regelungen nutzen
}

\section{Unternehmenspolitik in Zeiten des demografischen Wandels}

\author{
ECKHARD EYER \\ Eckhard Eyer ist Inhaber der \\ Perspektive Eyer Consulting in \\ Köln. Als Vergütungsberater und \\ Wirtschaftsmediator berät er \\ Einrichtungen und Verbände der \\ Wohlfahrtspflege bei der Gestal- \\ tung und Einführung von be- \\ trieblichen Entgeltsystemen und \\ der Leistungsvergütung sowie bei \\ (Haus-) Tarifverträgen. \\ www.eyer.de
}

\author{
Der »demografische Wandel« hat die Öffentlichkeit \\ und die Politik erreicht und vor einigen Jahren schon \\ die Tarif- und Unternehmenspolitik. Schwerpunkte \\ von Strategien zur Bewältigung des veränderten \\ Altersaufbaus im Unternehmen sind zum einen die \\ Förderung der Arbeitsfähigkeit älterer Menschen und \\ zum anderen die Gewinnung zusätzlicher Arbeitskräfte.
}

Wird die Entwicklung der Leistungsfähigkeit älterer Menschen diskutiert, so gehen die Akteure - je nach Erfahrungshintergrund, Branche und den $\mathrm{zu}$ betrachtenden Tätigkeiten - von einem Defizitmodell oder von einem Kompetenzmodell aus.

Das sogenannte Defizitmodell besagt, dass mit zunehmendem Alter die individuellen Leistungspotenziale, so auch die Innovationsfähigkeit der Menschen nachlassen. Im Rahmen des Defizitmodells werden Alterszuschreibungen wie geringerer Nutzen, eingeschränkte Gesundheit sowie häufigeres Vorkommen chronischer Erkrankungen angeführt.

Demgegenüber spricht das Kompetenzmodell den Älteren auch oberhalb der Altersgrenze von 50 Jahren Innovationspotenzial zu, sofern die Rahmenbedingungen entsprechend ausgestaltet sind. Das Kompetenzmodell beruht im Gegensatz zum Defizitmodell auf positiven Altersbildern. Es wird beispielsweise betont, dass der Bereich der sozialen Kompetenzen bei älteren Beschäftigten deutlich ausgeprägter sei als bei Jüngeren. Weiterhin werden ihnen ein höheres Erfahrungswissen sowie eine größere Zuverlässigkeit zugeschrieben.

Beide Modelle neigen - wie alle Modelle - zu Verallgemeinerungen und Stereotypen, da sie individuelle Abweichungen nicht berücksichtigen und die Kontextabhängigkeit von Fähigkeiten ausblenden. Unabhängig davon, welchem Denkmodell man zuneigt, stellt sich auf mindestens drei Gestaltungs- ebenen, die Frage, wie der demografische Wandel in Deutschland bewältigt werden soll: auf der gesellschaftlichen Ebene, auf der Ebene der Tarifpartner und auf der Ebene der Betriebspartner.

\section{Gesellschaft und demografischer Wandel}

Der demografische Wandel ist in der Gesellschaft angekommen. Schlagwörter sind Rente mit 67 oder 69 Jahren, Perspektive 50plus, Weiterbildung älterer Mitarbeiter, Gesundheitsförderung, Vereinbarkeit von Beruf und Familie, Blue Card für Menschen aus Drittstaaten und eine Arbeitserlaubnis für langjährig in Deutschland lebende Ausländer.

Die gesellschaftlichen Strategien hinter diesen Maßnahmen, um in Zukunft die notwendigen Arbeitskräfte und den Wohlstand in Deutschland zu sichern, heißen: Fördern der Beschäftigungsfähigkeit älterer Mitarbeiter, Erschließen der Stillen Reserve und Gewinnung von qualifizierten ausländischen Mitarbeitern. Dabei geht man auf der gesellschaftlichen Ebene - nicht zuletzt aus Gründen der Political Correctness - praktisch nur von dem Kompetenzmodell aus.

Neben den gesellschaftlichen Gruppen und politischen Parteien haben auch die Tarifvertragsparteien die Herausforderung erkannt und Tarifverträge abgeschlossen, die den demografischen Wandel aufgreifen. Beispielhaft seien hier mögliche Handlungsfelder der Sozialpartner skizziert und durch praktische Beispiele belegt. 


\section{Erhaltung der \\ Beschäftigungsfähigkeit älterer Mitarbeiter}

Aufgrund der aktuellen Herausforderungen bei der Personaleinsatzplanung von »leistungsgewandelten Mitarbeitern « haben die Sozialpartner sich des Themas des demografischen Wandels bereits vor zehn Jahren angenommen. Es waren Unternehmen und Branchen mit industrieller Produktion und einer ausgeprägten Schichtarbeit, die den Anfang machten. Seit Mitte des ersten Jahrzehnts dieses Jahrhunderts wurden erste Tarifverträge zur Demografie abgeschlossen und schrittweise weiterentwickelt.

Diese Tarifpartner gingen offensichtlich vom Defizitmodell aus. Sie wollten die Beschäftigungsfähigkeit der älteren Mitarbeiter erhalten, um auch künftig die qualitativen und quantitativen Herausforderungen am deutschen Arbeitsmarkt erfolgreich annehmen zu können. Die Sozialpartner haben eine Vielzahl verschiedener Handlungsoptionen, die auch kombinierbar sind: Sicherung der Beschäftigungsfähigkeit der älteren Mitarbeiter durch Gesundheitsförderung und Qualifizierung, angemessene Vergütung der Mitarbeiter zur Sicherung ihrer Beschäftigung sowie Frühverrentung von einsatz- und leistungsgeminderten Mitarbeitern.

\section{Erschließung der "Stillen Reserve"}

In der volkswirtschaftlichen Statistik tauchen in der "Stillen Reserve " Menschen auf, die arbeitsfähig und arbeitswillig sind, denen es aber aufgrund der aktuellen Lebensbedingungen nicht möglich ist, eine Arbeit anzunehmen; sie melden sich deshalb nicht bei der Agentur für Arbeit als arbeitsuchend. Menschen in der Stillen Reserve sind beispielsweise Menschen, die ihre Berufstätigkeit nicht mit der Kindererziehung oder der Pflege älterer Angehöriger verbinden können oder die an ihrem Wohnort keine Tätigkeiten finden und für die die Fahrt zu einem weit entfernten Arbeitsplatz ökonomisch nicht sinnvoll ist.

Um diese Menschen zu erreichen und sie für den Arbeitsmarkt zu gewinnen, bedarf es besonderer, auf sie zugeschnittener Angebote, deren Rahmen sich durch die Tarifvertragsparteien beispielsweise so gestalten lässt, dass sie aufgrund räumlicher und zeitlicher Flexibilität für diese Menschen attraktiv sind.

\section{Gewinnung von Menschen aus Drittstaaten}

Die Gewinnung von ausländischen Mitarbeitern aus Drittstaaten ist eine weitere Möglichkeit, die Lücke am Arbeitsmarkt zu schließen. Hier gibt es zwei grundlegende Strategien, zum einen können qualifizierte Mitarbeiter, die bereits in Deutschland leben und keine Arbeitserlaubnis haben, eine solche erhalten; zum anderen können Mitarbeiter im Ausland, die über entsprechende Qualifikationen verfügen, angeworben werden, um die Lücke am Arbeitsmarkt zu schließen.

\section{Was Unternehmen tun können}

Die Unternehmen - auch die der Sozialwirtschaft - müssen sich auf den Arbeitskräftemangel einstellen. Sie haben mehrere Grundstrategien, um ihn zu bewältigen. Dabei sind sie - im Gegensatz zu der gesellschaftlichen Ebene und der Ebene der Tarifpartner - grundsätzlich nicht an die nationalen Grenzen gebunden.

- Handlungsfeld Arbeitspolitik: Die Altersforschung konnte zeigen, dass sich Beschäftigte derselben Altersgruppe deutlich in ihren Fähigkeiten und ihren Leistungsmöglichkeiten unterscheiden. Unterschiede zwischen gleichaltrigen Mitarbeiterinnen und Mitarbeitern nehmen mit dem Alter tendenziell zu. Je nach Unternehmen und Arbeitsplatz sind sie mit unter-
Arbeitszeitgestaltung liegen die Gestaltungsmöglichkeiten bei dem Volumen, der Dauer und der Lage der Arbeitszeit. Durch entsprechende Veränderungen mit dem Ziel, die Belastungen der älteren Mitarbeiter zu verringern und so einen Beitrag zum Erhalt der Arbeitsfähigkeit leisten, kann das Unternehmen reagieren.

- Handlungsfeld Leistungspolitik: Basis für die Gestaltung der Arbeitsplätze sind zum einen die Auflagen der Berufsgenossenschaften und zum anderen nach Maßgabe des Betriebsverfassungsgesetzes die "gesicherten arbeitswissenschaftlichen Erkenntnisse über die menschengerechte Gestaltung der Arbeit«. Basis für die Bemessung der Leistung in den Unternehmen ist zusätzlich zum $\$ 611$ BGB die von den Sozialpartnern getragene - und von den Arbeitsgerichten genutzte - sogenannte »REFA-Normalleistung «. Sie geht von gewerblichen Tätigkeiten und Bewegungsabläufen aus. Der Arbeitgeberverband Südwestmetall und die IG Metall haben im Jahr 2003 im Entgeltrahmentarifvertrag (ERA-TV) eine tarifliche Bezugsleistung formuliert, die auf der REFA-Normalleistung basiert und sowohl für körperliche als auch für geistige Tätigkeiten gilt. Diese Formulierung wird weit über die vertragsschließenden Parteien hinaus als Norm akzeptiert. Gerade in der Sozialwirtschaft bedarf es bei der Dienstplangestaltung der Vorstellung einer Standardleistung, die Mitarbeiter erbringen, damit die an-

\section{"Jede Branche und jedes Unternehmen muss seinen eigenen Weg finden"}

schiedlichen Anforderungen konfrontiert. Es liegt daher nahe, im Kontext der jeweiligen Situation zu fragen: Welche Fähigkeiten liegen bei dem betreffenden Mitarbeiter vor und welche Kompensations- und Unterstützungsleistungen können durch den Betrieb erbracht werden? Möglichkeiten, den Arbeitsprozess dem einzelnen Mitarbeiter oder Mitarbeitergruppen anzupassen, liegen beispielsweise in der Arbeitsorganisation, der Arbeitsgestaltung und in den Regelungen, die die Arbeitszeit betreffen. Bei der liegende Arbeit fachlich und zeitlich zur Zufriedenheit der anvertrauten Menschen erledigt werden kann.

- Handlungsfeld Personalpolitik: Der Personalpolitik fällt im demografischen Wandel eine besondere Rolle zu, denn sie verfügt über ein breites Angebot an Interventionsmöglichkeiten: Rekrutierung, Personalentwicklung, Rentenübergang; Gestaltung von Teilzeitarbeit, Jobsharing, Home Office, schrittweise Pensionierung (Altersteilzeit); Arbeitszeitgestaltung zur besseren Vereinbarkeit von Familie und Be- 
Die Sozialpartner haben sich für unterschiedliche Handlungsfelder entschieden. Es zeigt sich, dass bei der Wahl der Strategien die jeweilige Problemlage der Branche entscheidend war. Dabei standen die Handlungsfelder "ältere Mitarbeiter" und "Stille Reserve" eindeutig im Vordergrund, Modellprojekte zur Beschäftigung ausländischer Mitarbeiter laufen an. Beispielhaft genannt ist hier als erstes ein Beispiel außerhalb der Sozialwirtschaft, das zeigt, dass jede Branche und jedes Unternehmen seine spezifischen Antworten finden muss:

- VW Tarifvertrag "Demografischer Wandel I«: Der Tarifvertrag aus dem Jahr 2007 stellt die gesundheitsförderliche und alternsgerechte Gestaltung der Arbeits- und Leistungsbedingungen, den Schutz der Gesundheit unter eigenverantwortlicher und weitestgehender Beteiligung der Beschäftigten, das Schaffen von Optionen für eine flexible und differenzierte Lebensarbeitszeit und das Sicherstellen einer möglichst ausgewogenen Altersstruktur in den Fokus und definiert als betriebliche Handlungsfelder die Unternehmenskultur, die Arbeitsgestaltung, das Gesundheitsmanagement, die Personal- und Teamentwicklung, die Personaleinsatzplanung und die Arbeitszeitgestaltung. Die Kosten der Maßnahmen trägt Volkswagen, also der Arbeitgeber.

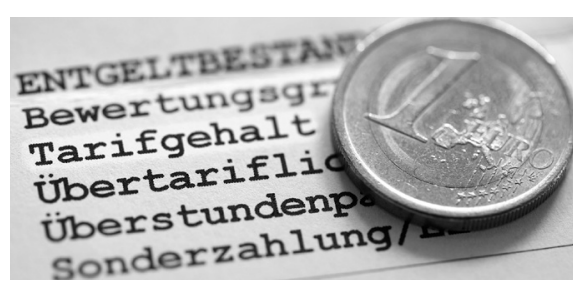

- Arbeitsvertragsrichtlinien der Caritas: In der Caritas ist der Anteil der beschäftigten Frauen im Vergleich zum produzierenden Gewerbe relativ hoch. Aus diesem Grund stand in den Verhandlungen der tarifsetzenden Arbeitsrechtlichen Kommission die Vereinbarkeit von Beruf und Familie im Vordergrund. Die Arbeitsvertragsrichtlinien der Caritas sehen seit 2011 eine Sozialkomponente zur besseren Vereinbarkeit von Beruf und Familie vor. Sie zielen somit insbesondere auf die Erschließung der "Stillen Reserve", beispielsweise durch die Finanzierung der Kinderbetreuung und den Erhalt der Arbeitsverhältnisse trotz der Pflege von Angehörigen ab. Die Kosten der Maßnahmen trägt der Arbeitgeber.

- Haustarifvertrag "Pflegen und Wohnen«: Das städtische Unternehmen der Freien und Hansestadt Hamburg "Pflegen und Wohnen" hat aufgrund der physisch und psychisch belastenden Arbeitsbedingungen in der Pflege der Gesundheitsförderung einen ho- hen Stellenwert beigemessen. In einem Haustarifvertrag mit ver.di wurde im Tarifvertrag ein Passus zur Gesundheitsförderung eingefügt ( $\$ 5 a)$, der festlegt, dass der Arbeitgeber ein jährliches Budget für Gesundheitsförderung zur Verfügung stellt. Die Höhe des Budgets beträgt für die circa 1.650 Mitarbeiter im Jahr 2012: 75.000 Euro, im Jahr 2013 100.000 Euro und im Jahr 2014 125.00 Euro.

- Arbeitgeberverband Pflege: Der Arbeitgeberverband Pflege, der bisher keine Tarifverträge abgeschlossen hat, setzt andere Akzente. Er hat mit den Regierungen von China und Deutschland seit Januar 2013 für die Dauer von fünf Jahren ein Modellprojekt laufen, bei dem 150 chinesische Mitarbeiter in Gruppen von 6 bis 10 Menschen in einer Einrichtung arbeiten. Die chinesischen Mitarbeiter sind fachlich hoch qualifiziert und wurden kulturell und sprachlich auf ihre Arbeit in den Altenund Pflegeheimen vorbereitet. Der Arbeitgeberverband Pflege verweist auf die guten Erfahrungen mit südostasiatischen Mitarbeitern in deutschen Krankenhäusern Ende der 196oer und Anfang der 1970er Jahre des letzten Jahrhunderts und die kulturbedingte Achtung der alten Menschen in den südostasiatischen Kulturkreisen.

Eckhard Eyer ruf; Nutzung gesetzlicher Regelungen, beispielsweise die betriebliche Ausgestaltung der Nutzung tariflicher Demografiefonds. Einen besonderen Stellenwert in der Personalpolitik haben die Angebote für jüngere potenzielle Mitarbeiter am Arbeitsmarkt und die Bindung der jüngeren Mitarbeiter an das Unternehmen. Die Rekrutierung und Weiterbildung sollte sich jedoch auch auf ältere Mitarbeiter beziehen, die über eine entsprechende Motivation und Fähigkeiten verfügen. Ihnen sind betriebliche Perspektiven - seien es Karrierechancen oder Rentenübergänge - zu eröffnen.

- Handlungsfeld Entgeltpolitik: Die Vergütung der Mitarbeiter in Deutschland folgt im Wesentlichen der Vorstellung, dass die Mitarbeiter nach dem Wert ihrer Arbeit vergütet werden. Dieser hängt zum einen davon ab, welche Anforderungen die Tätigkeit an den Mitarbeiter stellt und zum anderen, wie hoch das erarbei- tete Leistungsergebnis ist (doppeltes Äquivalenzprinzip von Kosiol). Legt man dieses Prinzip zugrunde, dann wird sich das Entgelt der Mitarbeiter entsprechend ihrer Einsatzfähigkeit und Leistungsfähigkeit entwickeln.

\section{Fazit}

Der demografische Wandel hat die Bundesrepublik Deutschland erreicht und bedroht die internationale Wettbewerbsfähigkeit des Standortes und die Wettbewerbsfähigkeit der Unternehmen.

Die Sozialwirtschaft kann aufgrund der notwendigen Kundennähe nur bedingt ihren Standort wählen und muss deshalb andere Antworten finden als das produzierende Gewerbe. Auf der gesellschaftlichen Ebene und der Ebene der Sozial- und Tarifpartner wurden erste symbolische nationale Anstrengungen unternommen, um den Standort zu stärken und den Wohlstand langfristig zu sichern.
Die Unternehmen müssen ihre Arbeits- und Leistungspolitik sowie Personal- und Entgeltpolitik auf die künftigen Gestaltungsmöglichkeiten hin ausloten. Aufgrund der Analyse und Bewertung der Situation ist ein optimaler StrategieMix zu entwickeln und umzusetzen. Hierbei kommt es in besonderem Maß auf die konstruktive Zusammenarbeit von Geschäftsleitung und Betriebsrat - aber auch der Sozialpartner - zur Sicherung der Wettbewerbsfähigkeit, des Standortes und der Arbeitsplätze im demografischen Wandel an.

\section{Literatur}

Eyer, E.: Demografie und Vergütung In: Industrial Engineering 2/2012. S. 22-25. Eyer, E.: Entgeltstrategien im demografischen Wandel. In Arbeit und Arbeitsrecht 2/2013.

Seite 110-113.

Eyer, E.: Sozialkomponente fördert Familie und Beruf. In Neue Caritas 5/2012. S. 22-25. 